\title{
Brain-Stem Relays Mediating Stimulation-Produced Antinociception from the Lateral Hypothalamus in the Rat
}

\author{
L. D. Aimone, ${ }^{a}$ C. A. Bauer, and G. F. Gebhart \\ Department of Pharmacology, College of Medicine, University of lowa, lowa City, lowa 52242
}

\begin{abstract}
Several lines of evidence have demonstrated a role for the lateral hypothalamus (LH) in an endogenous system of descending inhibition. The present study, in rats lightly anesthetized with pentobarbital, was undertaken to examine systematically the organization in the brain stem of pathways mediating descending inhibition of the nociceptive tail flick (TF) reflex produced by focal electrical stimulation in the LH. The microinjection of lidocaine into the midbrain, dorsolateral pons, or medial medulla resulted in significant increases in stimulation thresholds in the LH for inhibition of the TF reflex $(89.1,67.4$, and $73.6 \%$, respectively). Selective lesions of cell bodies in the midbrain or medulla by the neurotoxin ibotenic acid also produced significant increases in stimulation thresholds in the LH for inhibition of the TF reflex (31.6 and $131.6 \%$, respectively), thus revealing relays in the periaqueductal gray and the nucleus raphe magnus located between the LH and the lumbar spinal cord. The failure of ibotenic acid to affect $\mathrm{LH}$-produced descending inhibition when microinjected into the dorsolateral pons, and the significant effect produced by lidocaine microinjected into the same area, implicates fibers of passage in the dorsolateral pons in descending inhibition of the TF reflex produced by focal electrical stimulation in the LH. The fluorescent dye Fast blue and HRP conjugated to wheat germ agglutinin were used to confirm that the area stimulated in the LH has reciprocal connections with the periaqueductal gray and nucleus raphe magnus.
\end{abstract}

Focal electrical stimulation in the di- and telencephalon, including the hypothalamus, has been shown to inhibit responses of spinal dorsal horn neurons to peripheral noxious stimuli (Carstens, 1982; Carstens et al., 1982, 1983; Yezierski et al., 1983). Stimulation-produced antinociception (SPA) also has been demonstrated from the hypothalamus (Black et al., 1972; Balagura and Ralph, 1973; Goodman and Holcombe, 1976; Rhodes and Liebeskind, 1978; Hardy, 1985). A role for the lateral hypothalamus (LH) in endogenous systems of antinociception has been suggested by studies in the rat (Cox and Valenstein, 1965; Balagura and Ralph, 1973; Carr and Uysal, 1985; Kawajiri and

\footnotetext{
Received July 27, 1987; revised Oct. 28, 1987; accepted Nov. 3, 1987.

We gratefully acknowledge the secretarial assistance of Teresa Fulton and the technical assistance of Michael Burcham. Naloxone was graciously supplied by Dupont Pharmaceuticals. This work was supported by DHHS awards DA 02879 and NS 19912. L.D.A. was supported by T32 GM 07069.

Correspondence should be addressed to G. F. Gebhart at the above address.

a Present address: Laboratory of Neurosurgery Research, Mayo Clinic, Rochester, MN 55905.

Copyright (C) 1988 Society for Neuroscience $0270-6474 / 88 / 072652-12 \$ 02.00 / 0$
}

Satoh, 1985; Aimone and Gebhart, 1987; Behbehani et al., 1988) and monkey (Black et al., 1972; Goodman and Holcombe, 1976).

The $\mathrm{LH}$, including the medial forebrain bundle (MFB), has been shown to possess both ascending and descending components (Wolf and Sutin, 1966; Millhouse, 1969; Conrad and Pfaff, 1976; Grofová et al., 1978; Saper et al., 1979; Takagi et al., 1980; Beitz, 1982; Berk and Finkelstein, 1982; Nieuwenhuys et al., 1982; Veening et al., 1982; Peschanski and Besson, 1984; Schwanzel-Fukuda et al., 1984; Hosoya, 1985; Lovick, 1985; Behbehani et al., 1988). Berk and Finkelstein (1982) demonstrated by autoradiographic methods that the LH sends projections caudally that travel to the brain stem in 3 distinct pathways. These efferent fiber tracts of the LH course to, as well as through, the midbrain, pons, and medulla. The hypothalamus, including the LH, was reported by Beitz (1982) to provide the largest descending input to the periaqueductal central gray of the rat. Several other studies have also demonstrated connections between the LH and periaqueductal gray (PAG) (Wolf and Sutin, 1966; Conrad and Pfaff, 1976; Grofová et al., 1978; Saper et al., 1979; Marchand and Hagino, 1983; Behbehani et al., 1988). The degeneration of fibers following lesions in the $\mathrm{LH}$ of the rat revealed both dorsal and ventral projections from the LH to the PAG (Wolf and Sutin, 1966). The labeling of fibers by HRP (Grofová et al., 1978; Marchand and Hagino, 1983) or Phaseolus vulgaris leucoagglutinin (Behbehani et al., 1988) and the autoradiographic localization of ${ }^{3} \mathrm{H}$-amino acids microinjected into the LH (Saper et al., 1979) also confirm that the PAG receives afferent projections from the LH. Structures in the pons, including the parabrachial nucleus and the locus coeruleus (Veening et al., 1987; Saper et al., 1979) and medulla, including the nucleus raphe magnus (NRM), the nucleus raphe pallidus, and the nucleus paragigantocellularis lateralis (Peschanski and Besson, 1984; Schwanzel-Fukuda et al., 1984; Hosoya, 1985; Lovick, 1985), also receive projections from the LH. In addition, the LH has been shown to send direct projections to the cervical, lumbar, and sacral spinal cord in the rat (Hosoya, 1980; Berk and Finkelstein, 1982; Schwanzel-Fukuda, 1984; Veening et al., 1987).

Using HRP, Takagi et al. (1980) found several brain-stem structures-including the midbrain PAG, dorsolateral pons, and medullary NRM-that send projections rostrally through the ipsilateral MFB to the forebrain. Thus, electrical stimulation in the LH could orthodromically or antidromically activate one or more of these brain-stem structures (e.g., see Willis et al., 1984). While it is known that the LH has reciprocal connections with structures in the midbrain, pons, and medulla involved in systems of descending spinal inhibition, it is not clear which, if any, of these areas are functionally important in the descending inhibition produced by focal electrical stimulation in the LH. 
The present study was undertaken to examine systematically the organization of pathways in the brain stem mediating descending inhibition of the nociceptive tail flick (TF) reflex produced by focal electrical stimulation in the LH. The local anesthetic, lidocaine, and the neurotoxin, ibotenic acid, were used to produce reversible, nonselective, or irreversible somata-selective intracerebral lesions. Portions of these data have been previously reported (Aimone et al., 1987).

\section{Materials and Methods}

Animals. All experiments were performed in adult male Sprague-Dawley albino rats (Biolabs Inc., St. Paul, MN) weighing between 300 and 450 $\mathrm{gm}$ on the day of surgery. Anesthesia for surgery was induced with 45 $\mathrm{mg} / \mathrm{kg}$ pentobarbital sodium (Nembutal) given intraperitoneally. A femoral vein and artery were cannulated and a craniotomy performed. Following surgery, wound margins were covered with a local anesthetic ointment and a light level of anesthesia (corneal and flexion reflexes present) was maintained thereafter by a continuous intravenous infusion of pentobarbital, 3-6 mg/kg/hr (Sandkühler and Gebhart, 1984a, b; Aimone and Gebhart, 1986, 1987; Jones and Gebhart, 1986a). Rats were warmed on a heating pad, and arterial blood pressure was monitored continuously.

Noxious stimulation. The nociceptive TF reflex was evoked by focused radiant heat $(4 \times 10 \mathrm{~mm}$ area) applied to the underside of the tail (D'Amour and Smith, 1941; Ness and Gebhart, 1986). Heat was applied randomly to 6-7 evenly spaced sites along the tail starting $2.5 \mathrm{~cm}$ from its distal end. A cut-off time of $7 \mathrm{sec}$ was used to minimize damage to the skin of the tail (see Ness and Gebhart, 1986). Inhibition of the TF reflex was operationally defined as a latency $\geq 7 \mathrm{sec}$. Following inhibition of the TF reflex by supraspinal focal electrical stimulation, a control TF always followed at the next interval. Noxious heat was applied at $2 \mathrm{~min}$ intervals; this paradigm produces stable baseline TF latencies for severa hours without damage to the tail (Sandkühler and Gebhart, 1984a, b; Aimone and Gebhart, 1986, 1987; Jones and Gebhart, 1986a).

Brain stimulation. In the majority of experiments, the incisor bar was set $3.3 \mathrm{~mm}$ below horizontal zero (Paxinos and Watson, 1982). In experiments involving the dorsolateral pons, the incisor bar was set 10.0 $\mathrm{mm}$ below the horizontal zero to avoid rupturing the transverse sinus. Guide cannulae of 26 gauge $(0.46 \mathrm{~mm} \mathrm{o.d}$.) stainless steel tubing were stereotaxically placed $2 \mathrm{~mm}$ dorsal to the intended goal to allow for both focal electrical stimulation and drug microinjection at the same intracerebral site.

Monopolar focal electrical brain stimulation of continuous $100 \mathrm{~Hz}$ constant current, $100 \mu \mathrm{sec}$ duration cathodal pulses was used throughout. Brain stimulation was started $10 \mathrm{sec}$ before and continued during heating of the tail until the TF was evoked or $7 \mathrm{sec}$ had elapsed. Monopolar stimulating electrodes of 34 gauge $(0.15 \mathrm{~mm}$ o.d.) insulated magnet wire (Belden, Geneva, IL) inserted through the guide cannulae were used in all experiments. Electrodes were cut to extend $2 \mathrm{~mm}$ beyond the tip of the guide cannula. Stimulation intensities were increased stepwise until the TF reflex was inhibited (intensities ranged from 20 to $200 \mu \mathrm{A}$ ) or nonantinociceptive effects were noted (increases in blood pressure or muscle flexion/extension). Stimulation intensities did not exceed $200 \mu \mathrm{A}$ to minimize spread of current (Ranck, 1975).

Intracerebral microinjections. Microinjections were made with a 33 gauge $(0.20 \mathrm{~mm}$ o.d.) injection cannula inserted into and extending 2 $\mathrm{mm}$ beyond the end of the guide cannula. The injectate was delivered over a $1 \mathrm{~min}$ period, and the injection cannula was left in place an additional $1 \mathrm{~min}$ to minimize spread of the injectate along the injection track. The progress of the microinjection was continuously monitored by observing the travel of an air bubble in a length of calibrated PE-10 tubing inserted between the injection cannula and a $5 \mu \mathrm{l}$ syringe. The following drugs and doses were microinjected in $0.5 \mu 1$ volumes: $4 \%$ lidocaine (Astra $\left.{ }^{\circledR}\right)$ and ibotenic acid (10 $\mu \mathrm{g}$, Sigma).

Histology. At the end of the experiment, rats were killed with an overdose of pentobarbital. Anodal electrolytic lesions $(500 \mu \mathrm{A} \mathrm{DC}, 3$ $\mathrm{sec}$ ) were made to allow for histological verification of the electrode tracks or stimulation/microinjection sites in cresyl violet stained coronal brain sections $(40 \mu \mathrm{m})$.

Statistical analysis. Statistical comparisons were made using Student's paired $t$ test or 1 -way analysis of variance with Dunnett's post hoc test; $p<0.05$ was considered significant. Data are presented as means \pm SEM.
Anatomical tracing. Animals were anesthetized with pentobarbital $(45 \mathrm{mg} / \mathrm{kg})$, placed in a stereotaxic instrument and a craniotomy performed for injection of Fast blue or horseradish peroxidase conjugated to wheat germ agglutinin (HRP-WGA) into the LH. The injector was left in place $20 \mathrm{~min}$ and removed. The wound margin was sutured closed and covered with a local anesthetic ointment, and the rat was allowed to recover.

Afferent projections to the $L H$. A 3\% solution of Fast blue (Sigma) in distilled water was prepared and mixed by vortex just prior to use. A total volume of $50 \mathrm{nl}$ of Fast blue was injected into the same site in 2 volumes of $25 \mathrm{nl}$ each. Injections were made over a $1 \mathrm{~min}$ period and were separated by $5 \mathrm{~min}$. Following a survival time of $3 \mathrm{~d}$, rats were deeply anesthetized, the chest cavity opened, and 1000 units of heparin injected directly into the heart. The brain was perfused transcardially through the ascending aorta with saline followed by $10 \%$ formalin, removed, and stored overnight in $10 \%$ formalin at $4{ }^{\circ} \mathrm{C}$, and cut on a microtome in $40 \mu \mathrm{m}$ coronal sections. Every third section was mounted on a gelatin-coated slide and dried overnight. Sections were viewed under a fluorescence microscope using epifluorescence at the excitation wavelength of $360 \mathrm{~nm}$. The midbrain, pons, and medulla were examined for fluorescence, and labeled sections were drawn on corresponding pages from the atlas of the rat brain (Paxinos and Watson, 1982).

Efferent projections from the LH. A $10 \%$ solution of HRP-WGA in dimethyl sulfoxide was prepared and mixed by vortex just prior to use. A total volume of $100 \mathrm{nl}$ HRP-WGA was injected in 2 vol of $50 \mathrm{nl}$ each. Injections were made over a $1 \mathrm{~min}$ period and scparatcd by 5 min. Following a survival time of $36 \mathrm{hr}$, rats were deeply anesthetized, the chest cavity opened, and 1000 units of heparin injected directly into the heart. The brain was perfused transcardially with saline, a paraformaldehyde-gluteraldehyde (P-G) solution and a P-G sucrose solution through the ascending aorta, removed, and stored overnight in the $\mathrm{P}-\mathrm{G}$ sucrose solution. The brains were sectioned $(40 \mu \mathrm{m})$ on a cryostat and every third section saved. Free-floating sections were reacted using the tetramethyl benzidine method (modified from Mesulam, 1978) and mounted on chrom-alum-coated slides. After drying overnight, the sections were counterstained with neutral red and viewed under dark-field optics.

\section{Results}

\section{Intracerebral administration of lidocaine}

Stimulation thresholds for inhibition of the TF reflex were determined in the LH and also in one of the following sites in different experiments: the midbrain, dorsolateral pons, or rostral, ventral medulla. Following the establishment of pretreatment stimulation thresholds for inhibition of the TF reflex, lidocaine ( $4 \%$ in $0.5 \mu \mathrm{l}$ ) was microinjected into either the midbrain, dorsolateral pons, or rostral, ventral medulla to produce reversible, nonselective local anesthetic blocks. Multiple microinjections of lidocaine (2-3 $0.5 \mu \mathrm{l}$ microinjections), each separated vertically by $1.0 \mathrm{~mm}$, were made into the midbrain, while in other experiments, only single microinjections were made into the pons or medulla. The effect of lidocaine on the stimulation threshold in the $\mathrm{LH}$, as well as on the stimulation threshold at the site of microinjection, was determined periodically following the local anesthetic block.

These experiments were carried out to determine if these supraspinal sites are involved in descending inhibition of the TF reflex produced by focal electrical stimulation in the $\mathrm{LH}$. The results are summarized in Table 1.

\section{Midbrain}

An example of how these experiments were performed is illustrated in Figure 1. In this experiment, lidocaine $(4 \%, 0.5 \mu 1)$ was microinjected first into a histologically verified site (Fig. $1 C$, site a), and its effect on the stimulation threshold for inhibition of the TF reflex in the LH was determined. Subsequently, lidocaine was microinjected into sites $b$ and $c$, producing 50 and $100 \%$ increases, respectively, in the stimulation threshold in the 

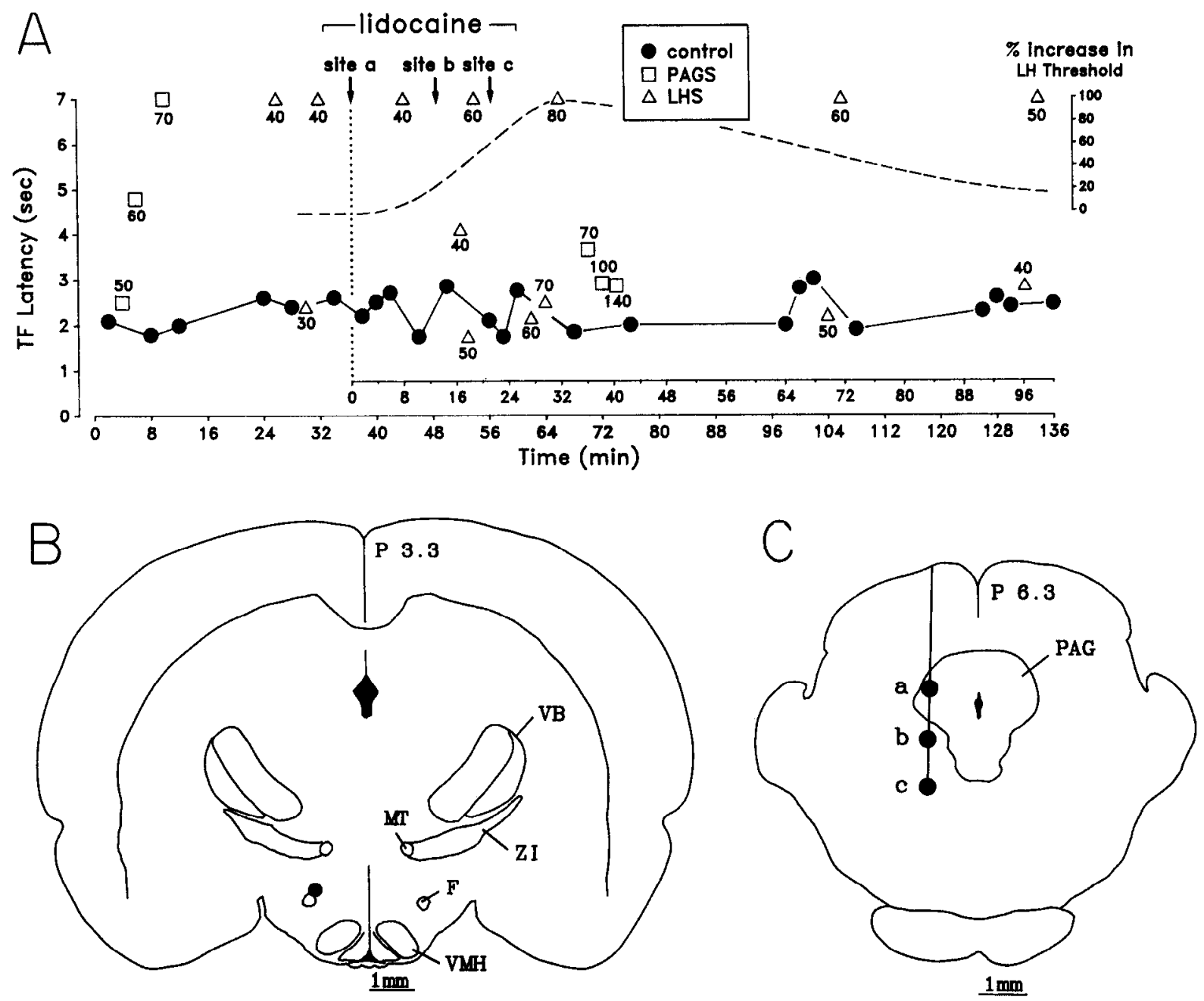

Figure 1. Example of the effects of lidocaine microinjected into the midbrain on stimulation thresholds in the LH for inhibition of the TF reflex. The TF latency $(\mathrm{sec})$ is plotted against time $(\mathrm{min})$; the times and sites of microinjections of lidocaine are indicated. Stimulation intensities for inhibition of the TF reflex (TF latency $\geq 7 \mathrm{sec}$ ) are given in $\mu \mathrm{A}$ (open squares, PAG stimulation $=$ PAGS; open triangles, LH stimulation $=$ LHS); TF latencies in the absence of stimulation are indicated by the filled symbols and are connected by a solid line. The broken line represents the change (\% increase) in the threshold of stimulation in the $\mathrm{LH}$ required to inhibit the TF reflex (right ordinate). The site of stimulation in the LH and of microinjections of lidocaine into the midbrain are indicated in $B$ and $C$, respectively.

\section{Table 1. Effect of microinjections of lidocaine or ibotenic acid on TF latency and lateral hypothalamic stimulation thresholds}

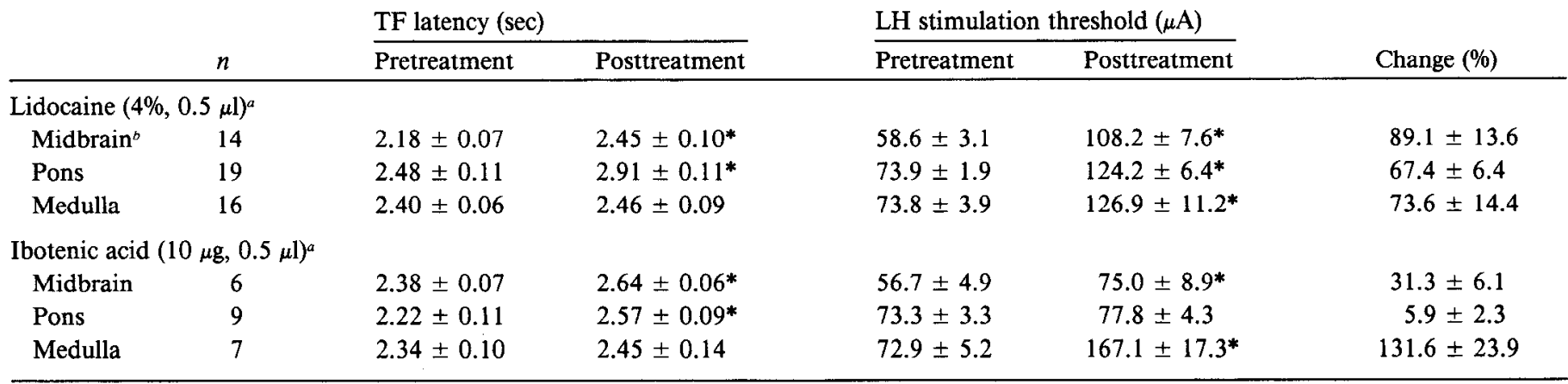

Data are reported as means \pm SEM. Significant differences $\left({ }^{*}\right)$, compared with pretreatment control, were determined using Student's paired $t$ test; $t$ values given in text $(p \leq 0.02$ in all cases).

${ }^{a}$ All intracerebral microinjections were made in a volume of $0.5 \mu \mathrm{l}$.

${ }^{b}$ Multiple microinjections of lidocaine were made into the midbrain; $n=14$ tracks, 40 injection sites (see text). 

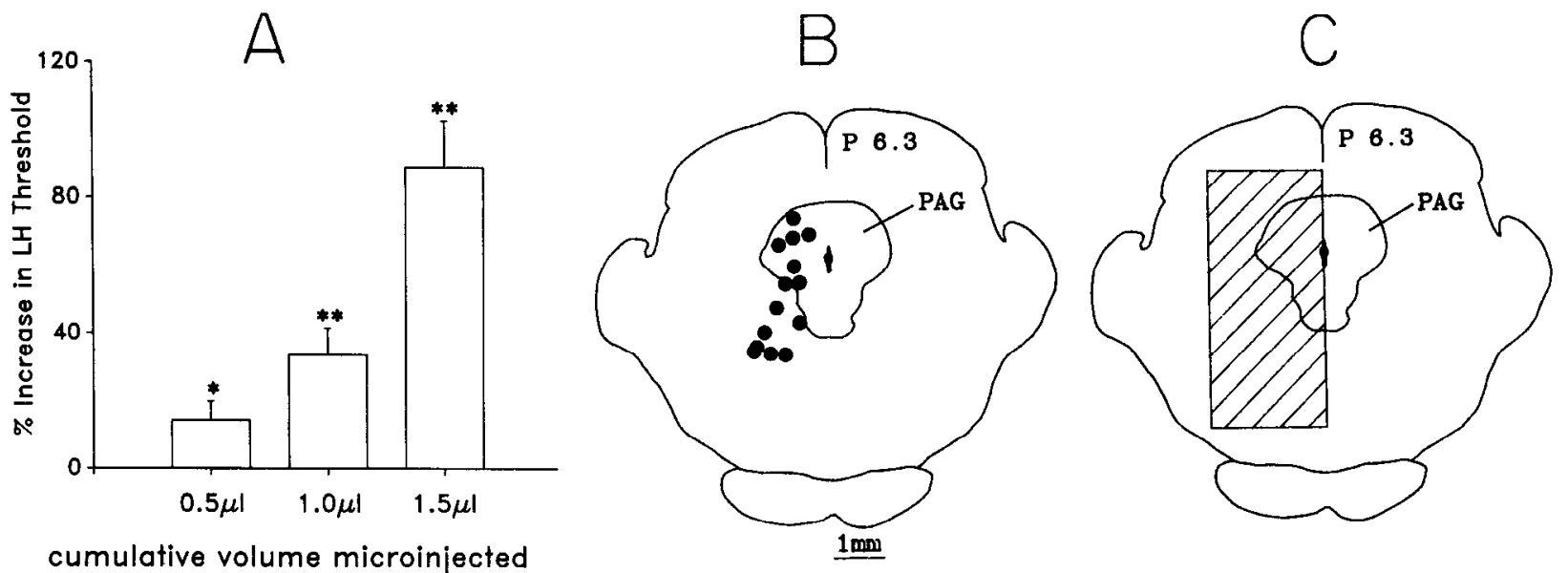

Figure 2. Summary of the effects of lidocaine microinjected into the midbrain on stimulation thresholds in the LH for inhibition of the TF reflex. $A$, Mean percentage increase $( \pm \mathrm{SEM})$ in the stimulation threshold in the LH following successive $0.5 \mu 1$ microinjections of lidocaine into the PAG. $B$, Closed circles $(n-14)$ represent the sites of the first microinjection of lidocaine into the PAG. $C$, Hatched area represents the estimated total area of the midbrain blocked following multiple microinjections of lidocaine.

LH for inhibition of the TF reflex. The efficacy of lidocaine was verified by the failure of stimulation in the midbrain (at twice the initial threshold) at the site of microinjection of lidocaine to inhibit the TF reflex. The data for 14 experiments are summarized in Figure 2.

The administration of a single microinjection $(0.5 \mu \mathrm{l})$ of lidocaine into the midbrain produced a small $(14.4 \pm 5.5 \%, n=$ $14)$, but statistically significant $(t=2.60, p \leq 0.05)$ increase in the stimulation threshold in the LH for inhibition of the TF reflex. Although distributed widely in the midbrain (Fig. $2 B$ ), there was no anatomical specificity apparent; microinjections of lidocaine into the midbrain either inside or outside the PAG resulted in a small increase in the stimulation threshold in the LH. In order to block a greater area of the midbrain, subsequent microinjections of lidocaine were made in the same experiment either $1 \mathrm{~mm}$ dorsal or $1 \mathrm{~mm}$ ventral to the initial site of microinjection (Fig. 3). Following the second microinjection of lidocaine, a greater increase $(33.4 \pm 8.1 \% ; t=3.88, p \leq 0.01)$ in the stimulation threshold in the LH was produced; an $89.1 \pm$ $13.6 \%(t=6.59, p \leq 0.001)$ increase in the stimulation threshold in the LH for inhibition of the TF reflex resulted following a third microinjection of lidocaine (Fig. $2 A$ ). A representation of the maximum area of midbrain functionally blocked by the multiple microinjections of lidocaine, estimated from studies in the brain stem and spinal cord (see Discussion), is illustrated in Figure $2 C$.

In all, 40 microinjections of lidocaine were made in 14 injection tracks through the midbrain in 14 rats. The sites of microinjection, all of which were ipsilateral to the stimulating electrode in the LH, and the magnitude of effect on the stimulation threshold in the $\mathrm{LH}$ are summarized in Figure 3. In all experiments $(n=14)$, the microinjection of lidocaine produced a greater than $100 \%$ increase in the stimulation threshold at the site of microinjection in the midbrain for inhibition of the TF reflex $(59.3 \pm 2.9$ to $128.5 \pm 5.7 \mu \mathrm{A} ; t=24.19, p \leq 0.001)$. This confirmed that the microinjection of lidocaine had produced a functional block. The effect of lidocaine in the midbrain and on the stimulation threshold in the LH was time-limited and reversible (see Fig. 1A). The control TF latency was slightly, but significantly, increased following the microinjection of li- docaine into the PAG $(2.18 \pm 0.07$ to $2.45 \pm 0.10 \mathrm{sec} ; t=3.24$, $p \leq 0.01$ ). Microinjection of lidocaine into the PAG did not produce significant changes in blood pressure.

\section{Dorsolateral pons}

The microinjection of lidocaine $(4 \%, 0.5 \mu \mathrm{l})$ into 19 sites in the dorsolateral pons ipsilateral to the stimulating electrode in the LH significantly increased the stimulation threshold in the $\mathrm{LH}$ for inhibition of the TF reflex $(73.9 \pm 1.9$ to $124.2 \pm 16.4 \mu \mathrm{A}$; $t=9.33, p \leq 0.001$ ). The stimulation threshold at these sites of microinjection of lidocaine in the dorsolateral pons also increased significantly $(133.3 \pm 4.0 \% ; t=21.13, p \leq 0.001)$ following the microinjection of lidocaine. These data are summarized in Figures 4 and 5. The majority of sites that produced significant increases in the stimulation threshold in the $\mathrm{LH}$ were located in and surrounding the locus coeruleus/subcoeruleus (LC/SC) (Fig. 4B). Microinjections of lidocaine into the cerebellum $(n=2)$ or into the ventral pons $(n=7)$ did not produce significant increases in the stimulation threshold in the LH for inhibition of the TF reflex. The control TF latency was significantly increased following microinjection of lidocaine into the dorsolateral pons $(2.48 \pm 0.11$ to $2.91 \pm 0.11 \mathrm{sec} ; t=3.53$, $p \leq 0.01$ ), but no changes in blood pressure were produced by lidocaine.

\section{Rostral, ventral medulla}

The microinjection of lidocaine $(4 \%, 0.5 \mu \mathrm{l})$ into 16 sitcs in the rostral, ventral medulla also significantly increased the stimulation threshold in the LH for inhibition of the TF reflex (73.8 \pm 3.9 to $126.9 \pm 11.2 \mu \mathrm{A} ; t=5.27, p \leq 0.001)$. The stimulation threshold at these sites of microinjection of lidocaine in the medulla also was increased significantly $(142.4 \% ; t=15.41, p \leq$ 0.001 ). These data are summarized in Figures 4 and 5 . The majority of sites that produced significant increases in the stimulation threshold in the $\mathrm{LH}$ were located in or just lateral to the NRM (see Fig. 4C). Lidocaine microinjections dorsal or further lateral to the NRM did not influence the stimulation threshold in the $\mathrm{LH}$ for inhibition of the TF reflex. Sites of microinjection of lidocaine $(n=7)$ adjacent to the NRM ipsilateral to the LH stimulating electrode produced increases in the LH stimulation 

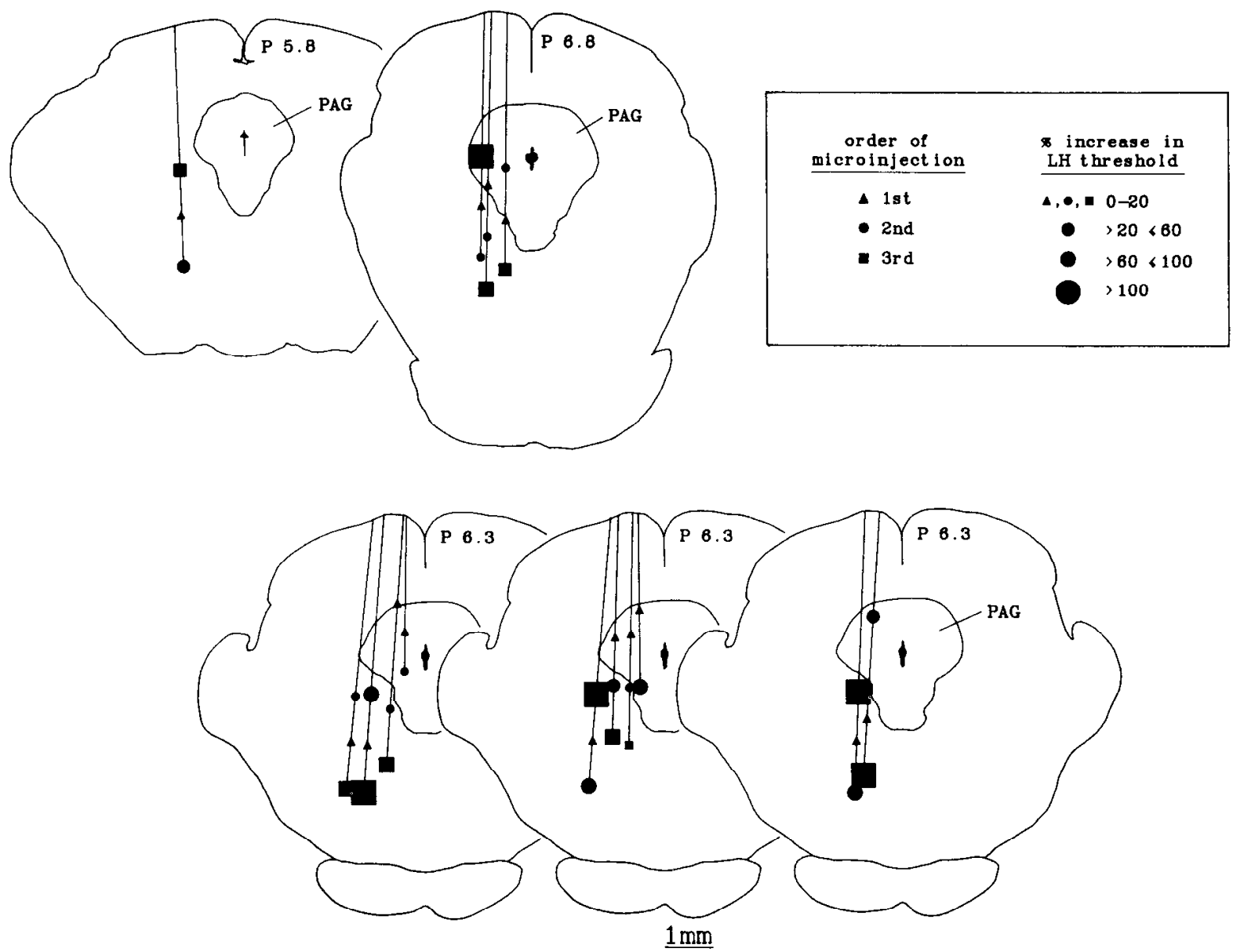

Figure 3. Histologic reconstructions of 14 tracks through, and 40 sites of microinjection of lidocaine into, the midbrain. Triangles, circles, and squares represent sites of the first, second, and third microinjection of lidocaine $(0.5 \mu \mathrm{l})$ into the midbrain in any one electrode track, respectively. The percentage increase in the stimulation threshold in the LH for inhibition of the TF reflex following each microinjection is represented by the varying symbol sizes (see inset), the largest symbol indicating a $>100 \%$ increase in the stimulation threshold in the LH for inhibition of the TF reflex.

threshold, while none $(0 / 5)$ of the sites located contralateral to the stimulating electrode in the LH produced increases in the stimulation threshold in the LH for inhibition of the TF reflex. Neither the control TF latency nor blood pressure was affected following microinjection of lidocaine into the medulla.

\section{Intracerebral administration of ibotenic acid}

Using the same experimental protocol as described above, stimulation thresholds for inhibition of the TF reflex were determined in the $\mathrm{LH}$ and also in one of the following sites in different experiments: the midbrain PAG, the LC/SC in the dorsolateral pons, or the NRM located in the rostral, ventral medulla. Following the establishment of pretreatment stimulation thresholds in the $\mathrm{LH}$ and either the PAG, LC/SC, or NRM for inhibition of the TF reflex, a single microinjection of ibotenic acid $(10 \mu \mathrm{g}$ in $0.5 \mu \mathrm{l}$ ) was made into the midbrain, pons, or medulla to produce irreversible, somata-selective lesions. The area of tissue influenced by this volume and concentration of ibotenic acid was estimated postmortem (histologically) to be $1.0-1.5 \mathrm{~mm}$ in diameter in the coronal plane, but this estimate is imprecise inasmuch as these were acute experiments. The effect of ibotenic acid on the stimulation threshold in the $\mathrm{LH}$, as well as on the stimulation threshold at the site of microinjection of ibotenic acid, was determined $1 \mathrm{hr}$ later. The microinjection of ibotenic acid produced decreases in blood pressure when microinjected into the midbrain, dorsolateral pons, and medial medulla. In all experiments, blood pressure had returned to control values by the time of testing $1 \mathrm{hr}$ later.

These experiments were carried out to determine if the increases in stimulation thresholds in the $\mathrm{LH}$ seen following the microinjection of lidocaine into these brain-stem sites was due to an effect on cell bodies or on fibers of passage. These results are illustrated in Figure 6 and summarized in Table 1.

\section{Midbrain}

The microinjection of ibotenic acid into 6 sites in the midbrain PAG ipsilateral to the stimulating electrode in the $\mathrm{LH}$ significantly increased the stimulation threshold in the LH for inhibition of the TF reflex $(56.7 \pm 4.9$ to $75.0 \pm 8.9 \mu \Lambda ; t=$ $3.84, p \leq 0.02)$. The stimulation thresholds in the PAG for inhibition of the TF reflex were also increased significantly $1 \mathrm{hr}$ following the microinjection of ibotenic acid into the midbrain $(51.7 \pm 3.1$ to $94.2 \pm 11.7 \mu \mathrm{A} ; t=4.33, p \leq 0.01)$. The sites of microinjection of ibotenic acid into the PAG $(n=10)$ are shown in Figure $6 C$. In 4 experiments in which the microinjeclion of ibotenic acid was outside the PAG, an increase in the stimulation threshold in the LH was not seen. In 2 of these cases, the stimulation threshold in the PAG also remained unchanged.

The microinjection of the neurotoxin into the PAG also re- 


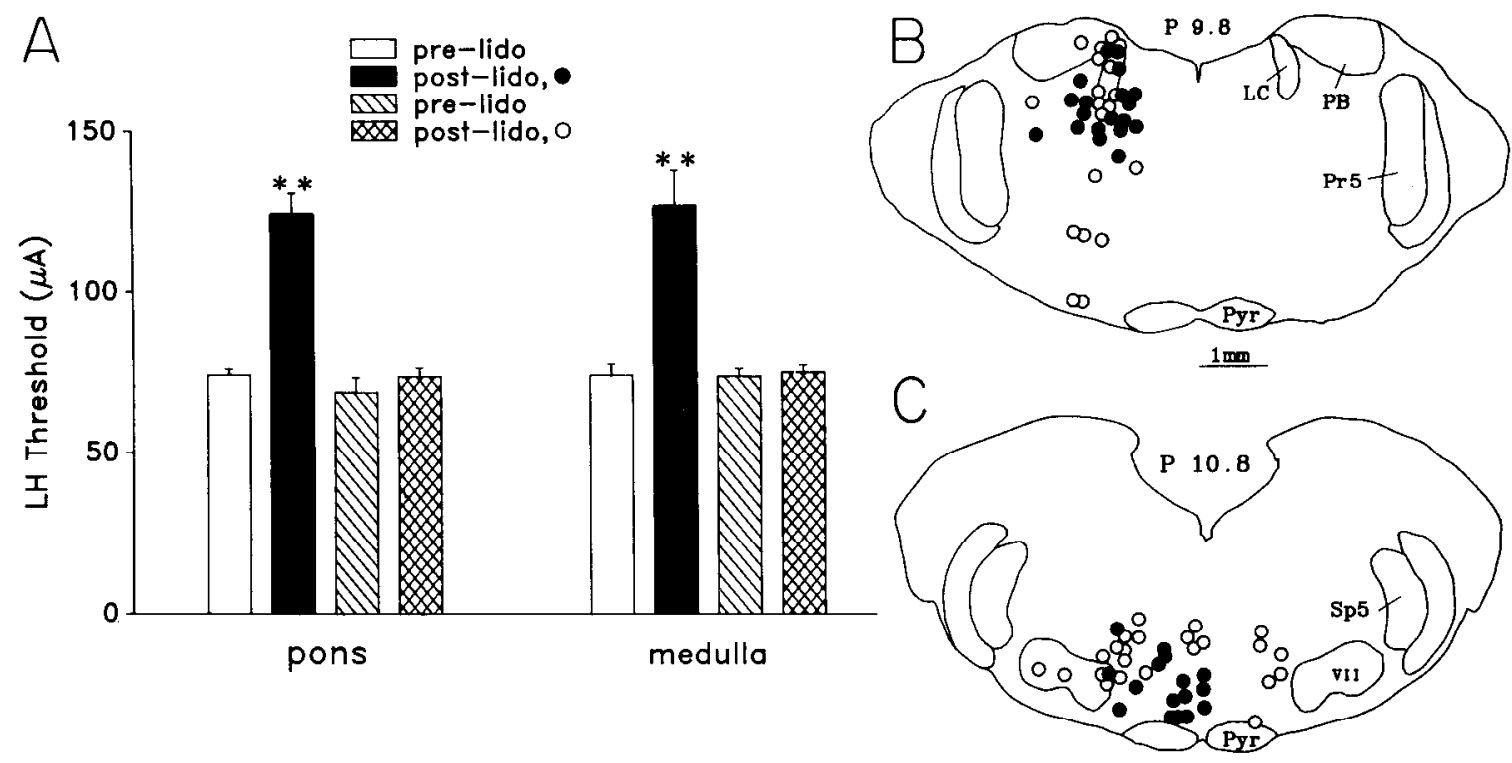

Figure 4. Summary of the effects of lidocaine microinjected into the pons or medulla on stimulation thresholds in the LH for inhibition of the TF reflex. $A$, Summary of stimulation thresholds in the $\mathrm{LH}$ following the microinjection of lidocaine into the pons or medulla. $B$ and $C$, Sites of microinjection of lidocaine into the pons or medulla, respectively. Closed circles represent sites at which lidocaine produced an increase in the stimulation threshold in the $\mathrm{LH}$; data from microinjections of lidocaine into these sites is represented by the flled bars in $A$. Open circles are sites at which lidocaine was ineffective; data from microinjections of lidocaine into these sites are represented by the cross-hatched bars in $A$. ${ }^{* *}$, Significantly different from the corresponding pre-lidocaine threshold $(p \leq 0.001)$.

sulted in a short-lived, reversible inhibition of the TF reflex in all experiments $(6 / 6)$. The mean duration of inhibition of the TF reflex was $12.5 \pm 3.7 \mathrm{~min}$. The inhibition of the TF reflex, in addition to the significant increase in stimulation thresholds at the site of microinjection and decreases in blood pressure $(12.0 \pm 4.1 \mathrm{~mm} \mathrm{Hg} ; l=2.90, p \leq 0.05)$, confirmed that the intracerebral administration of ibotenic acid was successful. In addition, as was seen following microinjections of lidocaine into the PAG, control TF latencies were also significantly increased $1 \mathrm{hr}$ after ibotenic acid microinjections into the PAG $(2.38 \pm$ 0.07 to $2.64 \pm 0.06 \mathrm{sec} ; t=3.98, p \leq 0.02$ ).

\section{Dorsolateral pons}

The microinjection of ibotenic acid into 12 sites in the pons ipsilateral to the stimulating electrode in the LH (Fig. 6B), 9 of which were in or adjacent to the LC/SC, did not affect stimu-

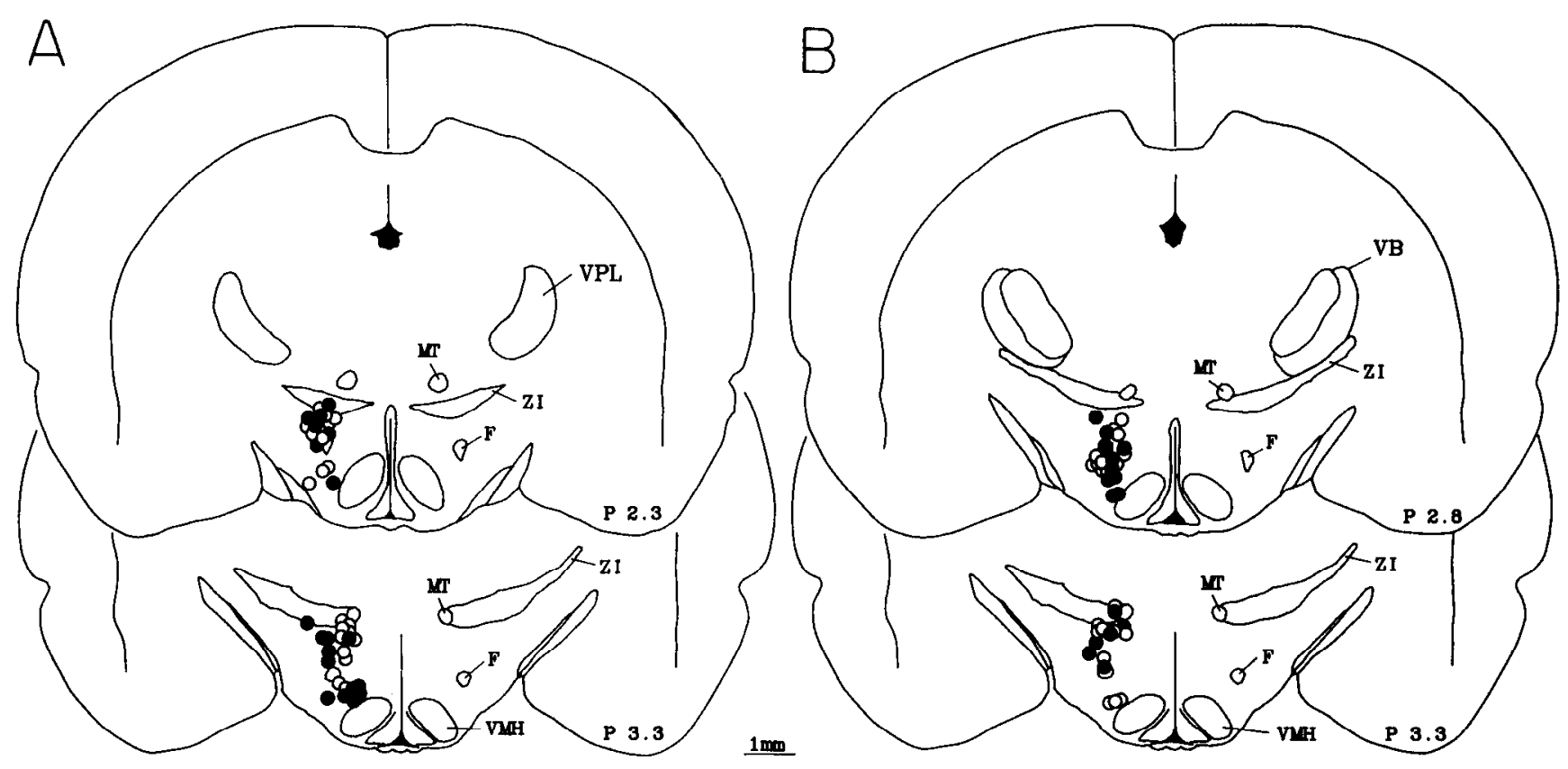

Figure 5. Sites of stimulation in the LII for inhibition of the TF reflex following the microinjection of lidocaine into the pons $(A)$ or medulla $(B)$. Closed circles represent sites where the stimulation threshold was increased following the microinjection of lidocaine into the pons or medulla. Open circles represent sites where the stimulation threshold was unchanged following microinjection of lidocaine into the pons or medulla. 

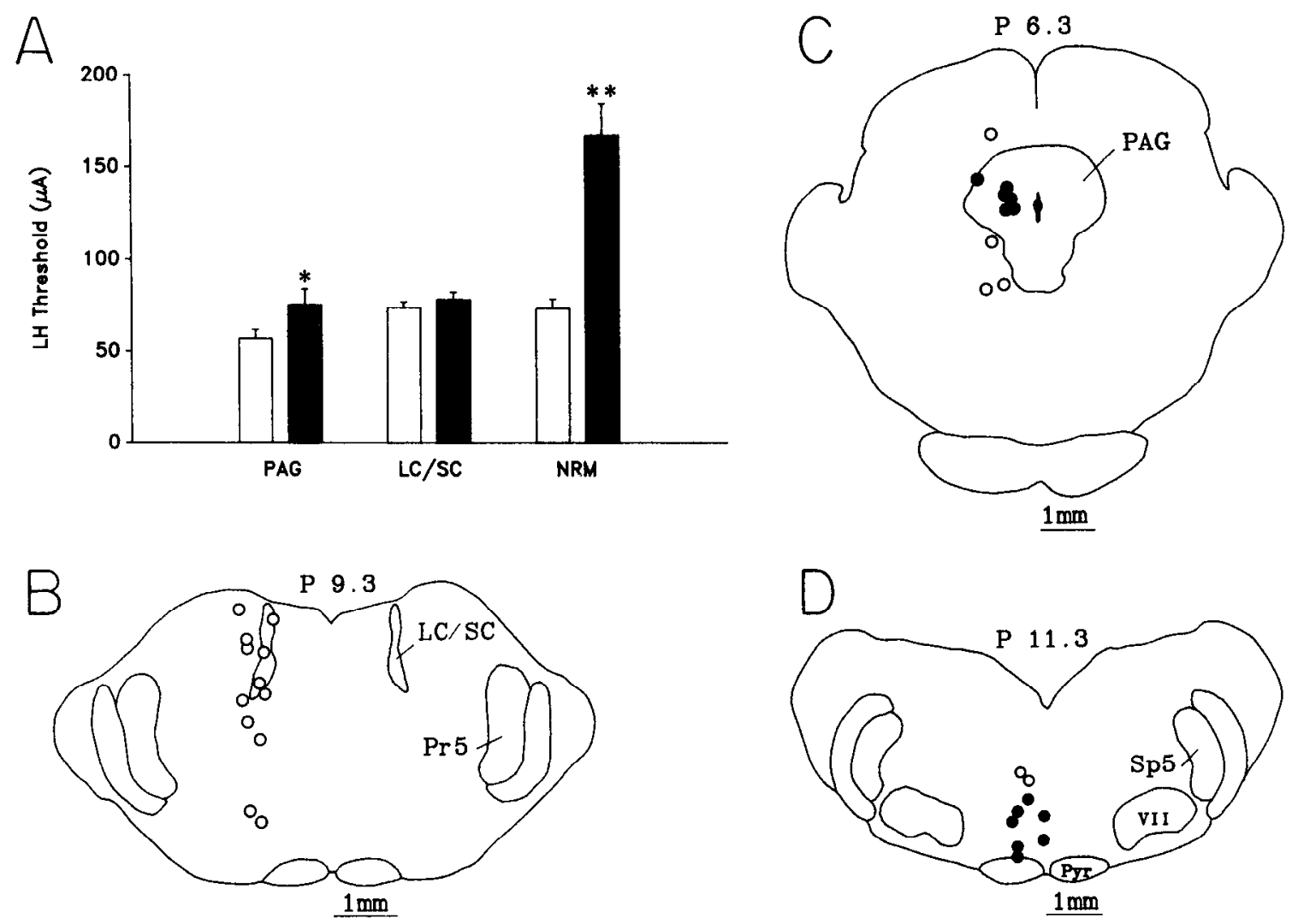

Figure 6. Summary of the effects of ibotenic acid microinjected into the midbrain, pons, or medulla on stimulation thresholds in the LH for inhibition of the TF reflex. $A$, Open bars represent the stimulation threshold in the LH before, and closed bars the LH threshold 1 hr after, microinjection of ibotenic acid into the midbrain, pons, or medulla. Sites of microinjection into the pons $(B)$, midbrain $(C)$, or medulla $(D)$ are shown. Closed circles represent sites at which ibotenic acid produced an increase in the stimulation threshold in the LH; open circles, sites at which ibotenic acid failed to affect the stimulation threshold in the LH. * and **, Significantly different from the corresponding pre-ibotenic acid threshold $(p \leq 0.01$ and 0.001 , respectively).

lation thresholds in the $\mathrm{LH}$ for inhibition of the TF reflex (73.3 \pm 3.3 to $77.8 \pm 4.3 \mu \mathrm{A}$ ). However, stimulation thresholds in the $\mathrm{LC} / \mathrm{SC}$ required to produce inhibition of the TF reflex were significantly increased $1 \mathrm{hr}$ following the microinjection of ibotenic acid $(32.2 \pm 2.2$ to $48.9 \pm 7.7 \mu \mathrm{A} ; t=2.43, p \leq 0.05$, $n=9$ ). The microinjection of the neurotoxin into or adjacent to the $\mathrm{LC} / \mathrm{SC}$ resulted in an inhibition of the TF reflex in 8 of 9 experiments. The mean duration of inhibition of the TF reflex was $11.9 \pm 3.6 \mathrm{~min}$, following which control TF latencies $1 \mathrm{hr}$ later were significantly increased compared with response latencies before the microinjection of ibotenic acid $(2.22 \pm 0.11$ to $2.57 \pm 0.09 \mathrm{sec} ; t=4.12, p \leq 0.01$ ). That the intracerebral administration of ibotenic acid was delivered was confirmed by inhibition of the TF reflex, as well as the increase in stimulation thresholds in the LC/SC for inhibition of the TF reflex and a significant decrease in blood pressure $(29.0 \pm 2.8 \mathrm{~mm} \mathrm{Hg} ;=$ $4.01, p \leq 0.005)$.

\section{Rostral, ventral medulla}

The microinjection of ibotenic acid into 7 sites in the medial medulla in or adjacent to the NRM significantly increased the stimulation threshold in the LH for inhibition of the TF reflex (72.9 \pm 5.2 to $167.1 \pm 17.3 \mu \mathrm{A} ; t=6.00, p \leq 0.001)$. Stimulation thresholds in the NRM for inhibition of the TF reflex were also increased significantly $1 \mathrm{hr}$ after the administration of ibotenic acid into the medial medulla $(24.0 \pm 3.8$ to $65.7 \pm 3.0 \mu \mathrm{A} ; t=$ $25.46, p \leq 0.001$ ), confirming the efficacy of the neurotoxin.
The sites of microinjection of ibotenic acid into the medulla $(n=9)$ are shown in Figure $6 D$. Consistent with what was observed following the microinjection of lidocaine into the rostral, ventral medulla, there was no significant change in control TF latencies following the microinjection of ibotenic acid, although in 3 experiments a short-lasting inhibition of the TF reflex was produced ( $3.7 \pm 0.7 \mathrm{~min})$. A decrease in blood pressure (11.0 \pm $3.6 \mathrm{~mm} \mathrm{Hg} ; n=7$ ) was also seen following the microinjection of ibotenic acid into the rostral, ventral medulla; however, this decrease was not statistically significant.

\section{Anatomical studies}

The retrogradely transported fluorescent dye Fast blue and HRPWGA were microinjected into the LH in different experiments to examine afferent and efferent projections, respectively, to and from sites in the LH where focal electrical stimulation could inhibit the TF reflex. The areas examined included the midbrain, the dorsolateral pons, and the rostral, ventral medulla; neither spinal cord nor forebrain areas were studied.

\section{Fast blue}

Three days after the microinjection of Fast blue $(3 \%, 50 \mathrm{nl})$ into the LH, the PAG, LC/SC, and NRM were examined for the presence of fluorescently labeled cell bodies. A reconstruction of the sites of injection of Fast blue $(n=3)$ is shown in Figure $7 A$. Of the 3 areas in the brain stem examined, the PAG contained the largest number of labeled cell bodies (Fig. 7B). La- 

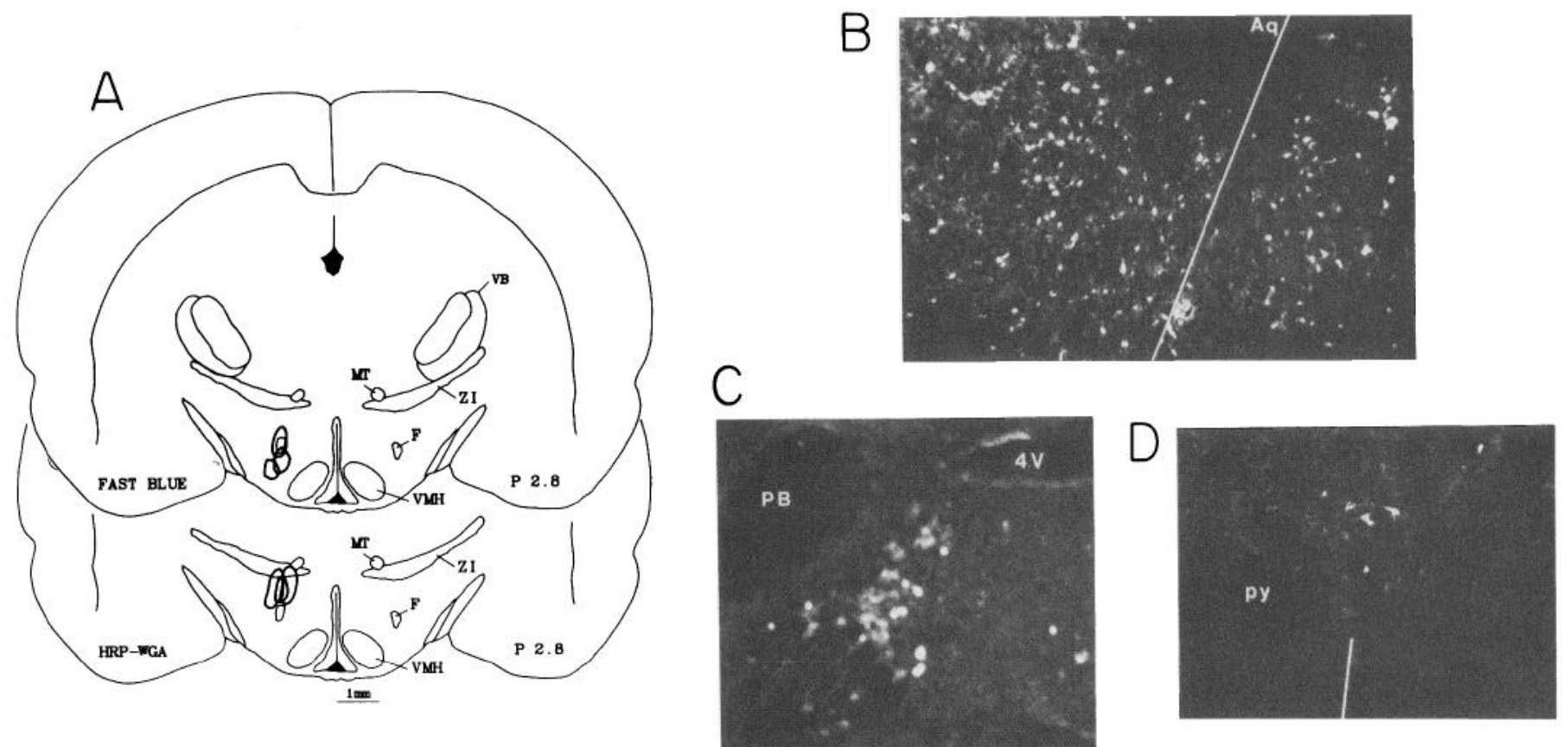

Figure 7. A, Reconstructed injection sites in the LH of Fast blue and HRP-WGA. Open symbols represent the centers of the sites of microinjection of Fast blue $(n=3)$ and HRP-WGA $(n=3)$ into the LH. These injection sites correspond to sites from which focal electrical stimulation produces an inhibition of the TF reflex. Photomicrographs of fluorescently labeled cell bodies in the midbrain $(B)$, pons $(C)$, and medulla $(D)$ following the microinjection of Fast blue into the LH. The lines in $B$ and $D$ have been added for orientation to the midline. Dorsal is top, and left is side ipsilateral to site of injection in the LH. $\times 80$. Abbreviations: $A q$, cerebral aqueduct; $P B$, parabrachial area; $P Y$, pyramid; and $4 V$, fourth ventricle.

beling was present and homogeneous in density throughout the PAG, although the labeled cell bodies were located predominantly ipsilateral to the site of injection in the LH. An area located ventral and lateral to the PAG also contained a dense cluster of fluorescently labeled cell bodies (see Fig. $8 B$ for location). Other midbrain nuclei containing labeled cell bodies included the dorsal raphe and the caudal linear raphe (Paxinos and Watson, 1982). Labeled cell bodies were also present in the LC but to a much lesser extent than in the midbrain (Fig. 7C). Labeling was also primarily ipsilateral to the site of injection in the LH. No labeled cell bodies were seen in the SC either ipsior contralateral to the site of microinjection of Fast blue. Other pontine structures that contained Fast blue-labeled cell bodies included the parabrachial nuclear complex, the Kölliker-fuse nucleus, the dorsal tegmental nucleus, and nucleus raphe pontis. The NRM was also labeled; however, filled cells were very sparse (Fig. 7D). The medulla also contained sparse labeling in the reticular formation adjacent and ipsilateral to the NRM and the caudal extent of the nucleus raphe pontis. The presence of cell bodies filled with Fast blue in the PAG, LC, and NRM confirms projections from these and other structures in the midbrain, pons, and rostral medulla to or through the LH.

\section{HRP-WGA}

Thirty-six hours after the microinjection of HRP-WGA $(10 \%$, $100 \mathrm{nl}$ ) into the $\mathrm{LH}$, the PAG, LC/SC, and NRM were examined for the presence of reaction product. A reconstruction of the sites of injection of HRP-WGA $(n=3)$ is shown in Figure $7 A$. Because HRP-WGA is transported in both the retro- and anterograde directions, 2 types of labeling were observed. Cell bodies filled with reaction product indicated retrograde transport, while punctuate labeling represented nerve terminals, revealing transport in the anterograde direction. In general, HRP-
WGA revealed a greater density of afferent projections to or through the $\mathrm{LH}$, as judged by the density of reaction product in cell bodies in the midbrain, pons, and medulla, than did Fast blue. The results, however, were highly complementary.

Two distinct efferent projections from the $\mathrm{LH}$ were seen in the midbrain, both of which terminated in the PAG. Projections reached the PAG in dorsal and ventral pathways. Within the PAG, dense labeling was seen in both cell bodies and terminals (Fig. 8A), emphasizing the reciprocal connection between the LH and PAG. As was observed following the microinjection of Fast blue into the $\mathrm{LH}$, reaction product in these cases was predominately ipsilateral to the site of microinjection of HRPWGA. With respect to the other midbrain structures retrogradely labeled with HRP-WGA, only cell bodies in the dorsal raphe nucleus and the caudal linear raphe nucleus contained reaction product, while reciprocal labeling was observed in the area located ventral and lateral to the PAG (Fig. $8 B$ ).

In the dorsolateral pons, reaction product was observed bilaterally in the LC but was predominantly ipsilateral (Fig. $8 \mathrm{C}$ ). Only cell bodies in the LC appeared to be labeled. Labeling of both cell bodies as well as nerve terminals was seen in the dorsal tegmental nucleus and in the parabrachial area. In the NRM, reaction product was seen in cell bodies and as punctuate labeling in nerve terminals (Fig. $8 D$ ). Reciprocal connections were also observed between the LH and the medullary reticular formation adjacent to the NRM.

\section{Discussion}

Inhibition of the spinal nociceptive TF reflex (Aimone and Gebhart, 1987) and spinal nociceptive transmission (Carstens et al., 1983) by electrical stimulation in the LH supports a role for the LH in system(s) of descending spinal inhibition. The present study considered contributions of sites in the brain stem also 

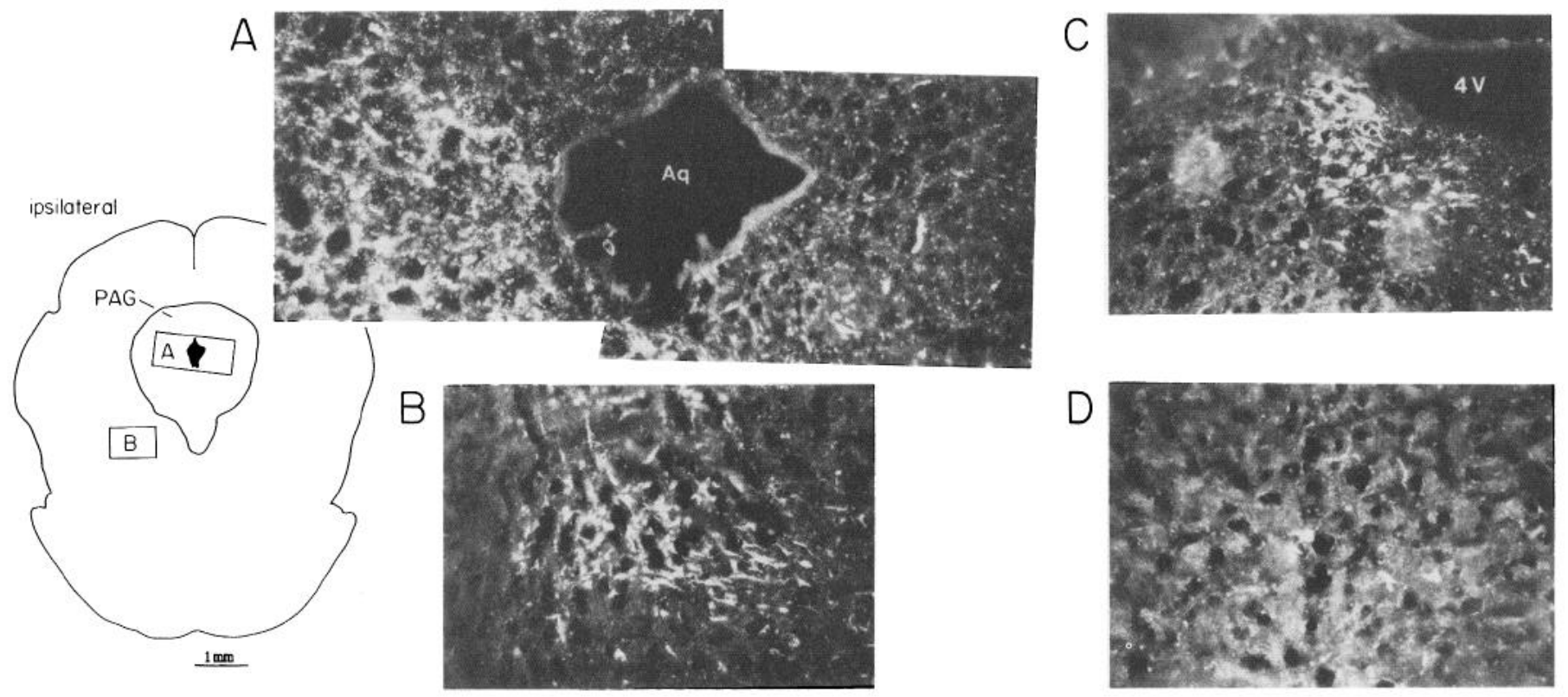

Figure 8. Dark-field photomicrographs of reaction product in the midbrain $(A$ and $B)$, pons $(C)$, and medulla $(D)$ following the microinjection of HRP-WGA into the LH. The approximate size and orientation of the photomicrographs in $A$ and $B$ are indicated in the adjacent section of the midbrain. Abbreviations and orientation as in Figure 7. $\times 160$.

implicated in systems of descending inhibition to the descending inhibition engaged in the LH, identifying areas in the midbrain, pons, and medulla which play a role in this descending inhibition from the LH. Selective lesions of cell bodies by the neurotoxin ibotenic acid revealed that cell bodies in both the midbrain PAG and medullary NRM can serve as relays between the $\mathrm{LH}$ and the spinal cord. An effect on descending inhibition from the LH by lidocaine microinjected into the dorsolateral pons, and the failure of ibotenic acid to reproduce the effect of lidocaine, suggests that a direct spinopetal pathway from the LH which passes through the dorsolateral pons may also contribute to descending inhibitory influences from the LH. Alternatively, the descending fibers from the $\mathrm{LH}$ that pass through the dorsolateral pons may terminate in the medial medulla. Anatomical tracing techniques were used to confirm that the LH receives afferent input from the PAG, $L C$, and NRM (among other structures) and, in addition, sends efferent projections to the PAG and NRM.

The use of lidocaine to produce a time-limited, reversible, functional block in the brain stem of the rat has been described by Sandkühler and Gebhart (1984b). They reported that the intramedullary microinjection of lidocaine $(0.5 \mu \mathrm{l}, 4 \%)$ produced a functional neural block having a radius of approximately 0.5 $\mathrm{mm}$ that was maximally efficacious during the first $30 \mathrm{~min}$ after the microinjection. These results have been confirmed (Aimone and Gebhart, 1986) and the efficacy of lidocaine for the production of reversible, central "lesions" has been well documented (e.g., Sandkühler and Gebhart, 1984b; Jones and Gebhart, 1987; Sandkühler et al., 1987). The microinjection of lidocaine in the present experiments into the midbrain, pons, or medulla resulted in significant increases in the stimulation threshold in the LH for inhibition of the TF reflex, indicating that the neuronal pathways which mediate descending inhibition from the LH pass through these areas.

In the midbrain, the administration of a single dose of lidocaine produced only a small $(14.4 \%)$ increase in the stimulation threshold in the LH for inhibition of the TF reflex, but subse- quent microinjections of lidocaine either dorsal and/or ventral to the original site of microinjection resulted in further increases (33.4 and $89.1 \%$ ) in the stimulation threshold in the LH. As indicated in Figure 3, the microinjection of lidocaine into an area ventrolateral to the PAG and dorsolateral to the interpeduncular nucleus appeared to be maximally efficacious in increasing the stimulation threshold in the LH. This area corresponds to the ventral pathway taken by descending fiber tracts from the LH to the PAG described by Wolf and Sutin (1966) and by Behbehani et al. (1988), using fiber degeneration methods and the lectin Phaseolus vulgaris leucoagglutinin as an anterograde tracer, respectively. This descending pathway from the LH also has been described by Berk and Finkelstein (1982) as the central tegmental tract. Descending fibers from the $\mathrm{LH}$ also reach the PAG via a dorsal periventricular pathway (Berk and Finkelstein, 1982; Behbehani et al., 1988). The results obtained in the present study using HRP-WGA also support the presence of both a dorsal and a ventral projection from the LH to the PAG. The ventral pathway was likely interrupted by the microinjections of lidocaine, which were made ventrolateral to the PAG. Thus, the results of these experiments are consistent with the relatively diffuse efferent projections from the LH to the PAG. The present data suggest that while both dorsal and ventral fiber bundles from the LH approach the PAG, the major portion of descending inhibitory influences from the area in the LH stimulated in this study is conveyed by the ventral fiber bundle (see Fig. 3). The neurotransmitter(s) in the midbrain mediating the antinociceptive effect of stimulation in the $\mathrm{LH}$ was not examined in this study, but Behbehani et al. (1988) suggest that neurotensin may be a (the) neurotransmitter of the dorsal fiber bundle projection from the LH to the PAG.

The dorsolateral pons and the rostral, ventral medulla were also implicated in the mediation of descending inhibition from the LH. Local anesthetic block of these areas with lidocaine also resulted in significant increases in stimulation thresholds in the LH for inhibition of the TF reflex. The LH has been shown to send projections to pontine and medullary reticular formations 
via the central tegmental tract, to the lateral dorsal tegmental nucleus of the pons via descending periventricular fibers, and to the NRM via the MFB (Berk and Finkelstein, 1982). The results of the present experiments are consistent with the location and interruption of these fiber tracts by the microinjection of lidocaine.

The specificity of the site of local anesthetic block is supported by the demonstration that not all sites of microinjections of lidocaine produced increases in the stimulation threshold in the LII. For example, microinjections of lidocaine into areas not identified by neuroanatomical tracing methods to contain a major descending fiber tract from the $\mathrm{LH}-$ such as the cerebellum, ventral pons, or lateral rostral, ventral medulla-did not produce increases in stimulation thresholds in the LH for inhibition of the TF reflex. Additionally, microinjections of lidocaine made into the rostral, ventral medulla contralateral to the stimulating electrode in LH did not affect stimulation thresholds in the LH, while microinjections just lateral to the NRM ipsilaterally did increase stimulation thresholds in the LH. This is consistent with reports that the majority of efferent projections from the LH are ipsilateral (Wolf and Sutin, 1966; Conrad and Pfaff, 1976; Saper et al., 1979; Berk and Finkelstein, 1982; SchwanzelFukuda et al., 1984; Behbehani et al., 1988). This was also confirmed in the present study.

Although the use of lidocaine established that areas in the midbrain, pons, and medulla may contribute to the descending inhibition arising from the $\mathrm{LH}$, lidocaine affects both cell bodies and fibers of passage, nonspecifically blocking neuronal conduction/transmission. In an attempt to resolve the relative contributions of cell somata and fibers of passage, the neurotoxin ibotenic acid, a conformationally restricted analog of the excitatory amino acid glutamate, was microinjected to selectively destroy neuronal somata. Schwarcz et al. (1979) examined the neurotoxic actions of ibotenic acid and found, at the light microscopic level, that axons of passage and nerve terminals of extrinsic origin were left intact while nerve cells had disappeared. Lesions were also restricted to the area of the microinjection, while lesions produced by kainic acid, for example, are less restricted (Schwarcz et al., 1979; Coyle and Schwarcz, 1983).

It has been postulated that the excitatory amino acids exert a neurotoxic effect because of their ability to act as agonists at glutamate receptors (McGeer and McGeer, 1981). These amino acids are thus termed "excitotoxins." This hypothesis is supported by the observation that microinjections of ibotenic acid into the PAG, LC/SC, or NRM produced a time-limited inhibition of the TF reflex in the majority of these experiments, presumably due to the excitation of cell bodies. The microinjection of glutamate into these areas has previously been demonstrated to produce short-lived inhibition of nociceptive responses (Behbehani and Fields, 1979; Urca et al., 1980; Satoh et al., 1983; Jensen and Yaksh, 1984; Aimone and Gebhart, 1986; Jones and Gebhart, 1986a) and of spinal dorsal horn neuronal responses to nociceptive stimuli (Jones and Gebhart, 1986b; Ness and Gebhart, 1987).

The microinjection of ibotenic acid into either the midbrain PAG or the medullary NRM produced significant increases in stimulation thresholds in the LH for inhibition of the TF reflex. Microinjections in the dorsolateral pons into and adjacent to the LC/SC did not, however, affect the stimulation threshold in the LH. From these results it can be concluded that cell bodies in the PAG and NRM, and fibers of passage through the dorsolateral pons, partially mediate and convey, respectively, de- scending inhibitory influences from the LH. However, as indicated above, LH efferents to the midbrain are diffuse and midbrain areas outside of the PAG also may be involved in the mediation of descending inhibition from the $\mathrm{LH}$. That ibotenic acid microinjected into the midbrain ventrolateral to the PAG failed to affect the stimulation threshold in the $\mathrm{LH}$ for inhibition of the TF reflex supports both the anatomical observations made here (and elsewhere) and results from experiments using lidocaine regarding the ventral course of an $\mathrm{LH}$ to PAG connection. Thus, it is likely that both cell bodies in the PAG, as well as fibers of passage through the midbrain, mediate descending inhibition from the LH. In addition to the relays in the PAG and NRM demonstrated here, inhibition of the TF reflex by stimulation in the LH may be mediated partly by direct spinopetal efferents from the LH to the lumbosacral spinal cord. Schwanzel-Fukuda et al. (1984) observed labeled cells in the lateral hypothalamus following the implantation of WGA into the cervical, lumbar, or sacral spinal cord of the rat. The pathway(s) in the brain stem taken by these descending fibers is not described, but it is possible that lidocaine microinjections in the present experiments interrupted these direct spinopetal efferents in either the dorsolateral pons or medial medulla.

The present study failed to detect the presence of anterograde labeling in the $\mathrm{LC} / \mathrm{SC}$ following the microinjection of HRPWGA into the LH. It has previously been reported that the LH scnds projections to thesc arcas (sce above). Howcver, AstonJones et al. (1987) recently demonstrated that afferents to the LC arise primarily from 2 areas in the rostral medulla. Following the microinjection of HRP-WGA into the LC, they observed consistent retrograde labeling only in the paragigantocellular nucleus and the prepositus hypoglossal nucleus. The apparent absence of afferent input from the LH to the LC is consistent with the failure of ibotenic acid microinjected into the LC to affect stimulation thresholds in the LH in the present study. The anatomical studies undertaken here were done primarily to confirm that sites of stimulation in the LH, effective in inhibition of the TF reflex, sent or received projections to or from areas in the brain stem documented as also contributing to the centrifugal modulation of nociception. As indicated previously, HRP-WGA revealed afferents to the LH more clearly than did Fast blue, but both methods gave complementary results. Both methods revealed afferents to the $\mathrm{LH}$ of the same relative magnitude; projections were most dense from the midbrain and least numerous from the rostral, ventral medulla. Similarly, projections from the LH to the PAG were extensive, while those to the rostral, ventral medulla were significantly less so. We detected virtually no projections from the $\mathrm{LH}$ to the LC/SC.

The foregoing, that both the PAG and NRM can apparently serve as relays between the $\mathrm{LH}$ and spinal cord, suggests that stimulation in the LH may activate the PAG, which in turn activates a bulbar relay in the NRM. This interpretation might appear to be contrary to the data obtained inasmuch as (1) we observed a relatively greater effect on stimulation thresholds in the LH by ibotenic acid microinjected into the NRM than produced by the same concentration and volume microinjected into the PAG, and (2) the effects of lidocaine microinjected into the NRM or PAG on stimulation thresholds in the LH were comparable. However, a significantly greater area of tissue was required to be blocked in the midbrain than in the medulla, consistent with the results of anatomical studies. As described by others (Wolf and Sutin, 1966; Conrad and Pfaff, 1976; Grofová et al., 1978; Saper et al., 1979; Beitz, 1982; Marchand and 
Hagino, 1983; Behbehani et al., 1988), and supported here, extensive efferents to the PAG arise from the LH. Electrophysiologically, Behbehani et al. (1988) demonstrated that electrical or chemical stimulation in the LH excites neurons in the PAG. They also suggest that neurotensin may be the neurotransmitter that mediates this effect. Beart et al.'(1987) provide autoradiographic anatomical as well as electrophysiological evidence that also supports the existence of an excitatory connection between the hypothalamus and PAG. An excitatory amino acid transmitter has also been implicated in this connection. Considerable anatomical and physiological evidence has documented that the NRM can serve as a bulbar relay between the PAG and spinal cord (see Aimone and Gebhart, 1986). Thus, a central LH $\rightarrow$ PAG $\rightarrow$ NRM descending inhibitory system is supported by converging lines of evidence.

Alternatively, descending inhibition from the LH mediated by the PAG need not necessarily involve the NRM. There are direct, albeit relatively few, spinopetal fibers from the PAG (Mantyh and Peschanski, 1982), and/or a relay in the rostral, ventral medulla lateral to the NRM may be involved (Beitz et al., 1983; Gebhart et al., 1983; Sandkühler and Gebhart, 1984b; Aimone and Gebhart, 1986). Further, mediation of descending inhibition from the $\mathrm{LH}$ via the NRM may be independent of any $\mathrm{LH} \rightarrow \mathrm{PAG}$ interaction. Direct connections from the $\mathrm{LH}$ to the medial medulla have been described (Peschanski and Besson, 1984; Schwanzel-Fukuda et al., 1984) and confirmed here. Thus, stimulation in the LH could simultaneously engage scveral independent descending systems of inhibition. An earlier examination of spinal monoaminergic mediation of descending inhibition from the LH supports the activation of multiple descending systems by stimulation in the LH (Aimone and Gebhart, 1987).

In addition, stimulation in the $\mathrm{LH}$ can also activate structures located rostral in the forebrain. Fibers to or from the forebrain are contained in the MFB and may be orthodromically or antidromically activated. Several brain-stem structures send projections through the ipsilateral MFB to the forebrain. Conversely, many forebrain structures send projections to the brain stem through the MFB. Hardy (1985) reported that stimulation in the prefrontal cortex (PFC) produced an increase in nociceptive response latencies in the hot plate and TF reflex tests. The PFC has also been shown to send projections to the midbrain PAG (Hardy and Leichnetz, 1981). Thus, stimulation in the LH also could activate a rostral loop, which, presumably via the PAG, may also contribute to descending inhibition from the $\mathrm{LH}$.

In summary, descending inhibition of the TF reflex produced by stimulation in the $\mathrm{LH}$ was shown in the present study to involve fibers of passage through the ventrolateral midbrain and the dorsolateral pons and/or neuronal relays in the midbrain PAG and the medullary NRM. The results of this study suggest the activation of multiple systems of descending inhibition by stimulation in the LH. The extent to which they may be independent or interdependent is unknown at present.

\section{References}

Aimone, L. D., and G. F. Gebhart (1986) Stimulation-produced spinal inhibition from the midbrain in the rat is mediated by an excitatory amino acid neurotransmitter in the medial medulla. J. Neurosci. 6: 1803-1813.

Aimone, L. D., and G. F. Gebhart (1987) Spinal monoamine mediation of stimulation-produced antinociception from the lateral hypothalamus. Brain Res. 403: 290-300.

Aimone, L. D., C. A. Bauer, and G. F. Gebhart (1987) Brainstem relays mediating stimulation-produced antinociception from the lateral hypothalamus. Soc. Neurosci. Abstr. 13: 115.

Aston-Jones, G., M. Ennis, V. A. Pieribone, W. T. Nickell, and M. T. Shipley (1987) The brain nucleus locus coeruleus: Restrictive afferent control of a broad efferent network. Science 234: 734-737.

Balagura, S., and T. Ralph (1973) The analgesic effect of electrical stimulation of the diencephalon and mesencephalon. Brain Res. 60: 369-379.

Beart, P. M., L. S. Nicolopolous, D. C. West, and P. M. Headley (1987) An excitatory amino acid projection from ventromedial hypothalamus (VMH) to periaqueductal gray (PAG) in the rat: Autoradiographic and electrophysiological evidence. Neurosci. Lett. 27: S54.

Behbehani, M. M., and H. L. Fields (1979) Evidence that an excitatory connection between the periaqueductal gray and the nucleus raphe magnus mediates stimulation produced analgesia. Brain Res. 170: 85-93.

Behbehani, M. M., M. R. Park, and M. E. Clement (1988) Interactions between the lateral hypothalamus and the periaqueductal gray. $J$. Neurosci. (in press).

Beitz, A. J. (1982) The organization of afferent projections to the midbrain periaqueductal gray of the rat. Neuroscience 7: 133-159.

Beitz, A. J., M. A. Mullet, and L. L. Weiner (1983) The periaqueductal projections to the rat spinal trigeminal, raphe magnus, gigantocellular pars alpha and paragigantocellular nuclei arise from separate neurons. Brain Res. 288: 307-314.

Berk, M. L., and J. A. Finkelstein (1982) Efferent connections of the lateral hypothalamic area of the rat: An autoradiographic investigation. Brain Res. Bull. 8: 511-526.

Black, P., S. N. Cianci, and R. S. Markowitz (1972) Alleviation of pain by hypothalamic stimulation in the monkey. Confin. Neurol. 34: 374-381.

Carr, K. D., and S. Uysal (1985) Evidence of a supraspinal opioid analgesic mechanism engaged by lateral hypothalamic electrical stimulation. Brain Res. 335: 55-62.

Carstens, E. (1982) Inhibition of spinal dorsal horn neuronal responses to noxious skin heating by medial hypothalamic stimulation in the cat. J. Neurophysiol. 48: 808-822.

Carstens, E., L. D. MacKinnon, and M. J. Guinan (1982) Inhibition of spinal dorsal horn neuronal responses to noxious skin heating by medial preoptic and septal stimulation in the cat. J. Neurophysiol. 48: 981-991.

Carstens, E., M. Fraunhoffer, and S. N. Suberg (1983) Inhibition of spinal dorsal horn neuronal responses to noxious skin heating by lateral hypothalamic stimulation in the cat. J. Neurophysiol. 50: 192204

Conrad, L. C. A., and D. W. Pfaff (1976) Efferents from medial basal forebrain and hypothalamus in the rat. J. Comp. Neurol. 169:221262.

Cox, V. C., and E. S. Valenstein (1965) Attenuation of aversive properties of peripheral shock by hypothalamic stimulation. Science 149 : 323-325.

Coyle, J. T., and R. Schwarcz (1983) The use of excitatory amino acids as selective neurotoxins. In Handbook of Chemical Neuroanatomy, Vol. I, Methods in Chemical Neuroanatomy, A. Björklund and T. Hökfelt, eds., pp. 508-527, Elsevier, Amsterdam.

D'Amour, F. E., and D. L. Smith (1941) A method for determining the loss of pain sensation. J. Pharmacol. Exp. Ther. 72: 74-79.

Gebhart, G. F., J. Sandkühler, J. G. Thalhammer, and M. Zimmermann (1983) Inhibition of spinal nociceptive information by stimulation in midbrain of the cat is blocked by lidocaine microinjections in nucleus raphe magnus and medullary reticular formation. J. Neurophysiol. 50: 1446-1459.

Goodman, S. J., and V. Holcombe (1976) Selective and prolonged analgesia in monkey resulting from brain stimulation. Adv. Pain Res. Ther. 1: 495-502.

Grofová, I., O. P. Ottersen, and E. Rinvik (1978) Mesencephalic and diencephalic afferents to the superior colliculus and periaqueductal gray substance demonstrated by retrograde axonal transport of horseradish peroxidase in the cat. Brain Res. 146: 205-220.

Hardy, S. G. P. (1985) Analgesia elicited by prefrontal stimulation. Brain Res. 339: 281-284.

Hardy, S. G. P., and G. R. Leichnetz (1981) Frontal cortical projections to the periaqueductal gray in the rat: A retrograde and orthograde horseradish peroxidase study. Neurosci. Lett. 23: 13-17.

Hosoya, Y. (1980) The distribution of spinal projection neurons in 
the hypothalamus of the rat, studied with the HRP-method. Exp. Brain Res. 40: 79-88.

Hosoya, Y. (1985) Hypothalamic projections to the ventral medulla oblongata in the rat, with special reference to the nucleus raphe pallidus: A study using autoradiographic and HRP techniques. Brain Res. 344: 338-350.

Jensen, T. S., and T. L. Yaksh (1984) Spinal monoamine and opioid systems partially mediate an analgesia produced by glutamate at brainstem sites. Brain Res. 321: 287-298.

Jones, S. L., and G. F. Gebhart (1986a) Characterization of coeruleospinal inhibition of the nociceptive tail-flick reflex in the rat: Mediation by spinal $\alpha_{2}$-adrenoceptors. Brain Res. 364: 315-330.

Jones, S. L., and G. F. Gebhart (1986b) Quantitative characterization of coeruleospinal inhibition of nociceptive transmission in the rat. $\mathrm{J}$. Neurophysiol. 56: 1397-1410.

Jones, S. L., and G. F. Gebhart (1987) Spinal pathways mediating tonic, coeruleospinal and raphespinal inhibition of nociceptive transmission in the rat. J. Neurophysiol. 58: 138-159.

Kawajiri, S.-I., and M. Satoh (1985) Analgesic effects of cyclazocine and morphine microinjected into the rat dorsomedial hypothalamus demonstrated by bradykinin-induced flexor reflex test. Eur. J. Pharmacol. 11: 117-120.

Lovick, T. $\Lambda$. (1985) Projections from the diencephalon and mesencephalon to nucleus paragigantocellularis lateralis in the cat. Neuroscience 14: 853-861.

Mantyh, P. W., and M. Peschanski (1982) Spinal projections from the periaqueductal gray and dorsal raphe in the rat, cat and monkey. Neuroscience 7:2769-2776.

Marchand, J. E., and N. Hagino (1983) Afferents to the periaqueductal gray in the rat. A horseradish peroxidase study. Neuroscience 9: 95106.

McGeer, E. G., and D. L. McGeer (1981) Neurotoxins as tools in neurobiology. Int. Rev. Neurobiol. 22: 173-204.

Mesulam, M.-M. (1978) Tetramethyl benzidine for horseradish peroxidase neurohistochemistry: A non-carcinogenic blue reaction-product with superior sensitivity for visualizing neural afferents and efferents. J. Histochem. Cytochem. 26: 106-117.

Millhouse, O. E. (1969) A Golgi study of the descending medial forebrain bundle. Brain Res. 15: 341-363.

Ness, T. J., and G. F. Gebhari (1986) Centrifugal modulation of the rat tail flick reflex evoked by graded noxious heating of the tail. Brain Res. 386: 41-52.

Ness, T. J., and G. F. Gebhart (1987) Quantitative comparison of inhibition of visceral and cutaneous spinal nociceptive transmission from the midbrain and medulla in the rat. J. Neurophysiol. 58: 18671892 .

Nieuwenhuys, R., L. M. G. Geeraedts, and J. G. Veening (1982) The medial forebrain bundle of the rat. I. General introduction. J. Comp. Neurol. 206: 49-81.

Paxinos, G., and C. Watson (1982) The Rat Brain in Stereotaxic Coordinates, $\Lambda$ cademic, New York.

Peschanski, M., and J.-M. Besson (1984) Diencephalic connections of the raphe nuclei of the rat brainstem: An autonomical study with reference to the somatatory system. J. Comp. Neurol. 224: 509-534.

Ranck, J. B., Jr. (1975) Which elements are excited in electrical stimulation of mammalian central nervous system: A review. Brain Res. 98: $417-440$
Khodes, D. L., and J. C. Liebeskind (1978) Analgesia from rostral brain stem stimulation in the rat. Brain Res. 143: 521-532.

Sandkühler, J., and G. F. Gebhart (1984a) Characterization of inhibition of a spinal nociceptive reflex by stimulation medially and laterally in the midbrain and medulla in the pentobarbital-anesthetized rat. Brain Res. 305: 67-76.

Sandkühler, J., and G. F. Gebhart (1984b) Relative contribution of the nucleus raphe magnus and adjacent medullary reticular formation to the inhibition by stimulation in the periaqueductal gray of a spinal nociceptive reflex in the pentobarbital-anesthetized rat. Brain Res. 305: 77-87.

Sandkühler, J., B. Maisch, and M. Zimmermann (1987) The use of local anesthetic microinjections to identify central pathways: A quantative evaluation of the time course and extent of the neuronal block. Exp. Brain Res. 68: 168-178.

Saper, C. B., L. W. Swanson, and W. M. Cowan (1979) An autoradiographic study of the efferent connections of the lateral hypothalamic area in the rat. J. Comp. Neurol. 183: 689-706.

Satoh, M., R. Oku, and A. Akaike (1983) Analgesia produced by microinjection of l-glutamate into the rostral ventromedial bulbar nuclei of the rat and its inhibition by intrathecal $\alpha$-adrenergic blocking agents. Brain Res. 261: 361-364.

Schwanzel-Fukuda, M., J. I. Morrell, and D. W. Pfaff (1984) Localization of forebrain neurons which project directly to the medulla and spinal cord of the rat by retrograde tracing with wheat germ agglutinin. J. Comp. Neurol. 266: 1-20.

Schwarcz, R., T. Hökfelt, K. Fuxe, C. Jonsson, M. Goldstein, and L. Terenius (1979) Ibotenic acid-induced neuronal degeneration: A morphological and neurochemical study. Exp. Brain Res. 37: 199216.

Takagi, H., S. Shiosaka, M. Tohyama, E. Senba, and M. Sakanaka (1980) Ascending components of the medial forebrain bundle from the lower brain stem in the rat, with special reference to raphe and catecholamine cell groups. A study by the HRP method. Brain Res. 193: 315-337.

Urca, G., R. L. Hin, and J. C. Liebeskind (1980) Glutamate-induced analgesia: Blockade and potentiation by naloxone. Brain Res. 192: 523-530.

Veening, J. G., L. W. Swanson, W. M. Cowan, R. Nieuwenhuys, and L. M. G. Geeraedts (1982) The medial forebrain bundle of the rat. II. An autoradiographic study of the topography of the major descending and ascending components. J. Comp. Neurol. 206: 82-108.

Veening, J. G., S. Te Lie, P. Posthuma, L. M. G. Geeraedts, and R. Nieuwenhuys (1987) A topographical analysis of the origin of some efferent projections from the lateral hypothalamic area in the rat. Neuroscience 22: 537-551.

Willis, W. D., K. D. Gerhart, S. W. Willcockson, R. P. Yezierski, T. L. Wilcox, and C. L. Cargill (1984) Primate raphe- and reticulospinal neurons: Effects of stimulation in periaqueductal gray or $\mathrm{VPL}_{\mathrm{c}}$ thalamic nucleus. J. Neurophysiol. 51: 467-480.

Wolf, G., and J. Sutin (1966) Fiber degeneration after lateral hypothalamic lesions in the rat. J. Comp. Neurol. 127: 137-156.

Yezierski, R. D., K. D. Gerhart, R. J. Schrock, and W. D. Willis (1983) A further examination of effects of cortical stimulation on primate spinothalamic tract cells. J. Neurophysiol. 49: 424-441. 